\title{
Factors Associated with Changes in Brain Atrophy during a Three-Year Observation in Elderly Diabetic Patients: Effect of Renal Impairment on Hippocampal Atrophy
}

\author{
Takahiko Kawamura ${ }^{a, c}$ Toshitaka Umemura ${ }^{b}$ Hiroyuki Umegakid ${ }^{d}$ Rui Imamine ${ }^{a}$ \\ Naoko Kawano ${ }^{\text {, e }}$ Hajime Mase $^{a}$ Asako Mizoguchi ${ }^{a}$ Makiko Minatoguchi ${ }^{a}$ \\ Minoru Kusama ${ }^{a}$ Yu Kouchia Atsuko Watarai ${ }^{c}$ Akio Kanaic Eitaro Nakashima ${ }^{a}$ \\ Nigishi Hotta ${ }^{a}$ \\ Departments of a Diabetes and Endocrine Internal Medicine and ${ }^{b}$ Neurology and \\ ${ }^{c}$ Center for Preventive Medicine, Chubu Rosai Hospital, and \\ Departments of ${ }^{\mathrm{d} C o m m u n i t y ~ H e a l t h ~ C a r e ~ a n d ~ G e r i a t r i c s ~ a n d ~}{ }^{\mathrm{e}}$ Psychiatry, Nagoya University \\ Graduate School of Medicine, Nagoya, Japan
}

\section{Key Words}

Brain atrophy · Cognitive impairment - Diabetes mellitus - Cerebral small vessel disease . Renal impairment

\section{Abstract}

Background/Aims: We conducted a 3-year longitudinal study concerning factors associated with changes in brain atrophy in elderly diabetic patients. Methods: We evaluated hippocampal and global brain atrophy using automatic voxel-based morphometry of structural magnetic resonance images, 4 cognitive function tests, and cerebral small vessel disease (SVD) in 66 diabetic patients. Results: During the 3-year follow-up, hippocampal and global brain atrophy advanced, and cognitive functions worsened. For changes in hippocampal atrophy, changes in estimated glomerular filtration rate (eGFR), albuminuria, and being an ApoE $\varepsilon 4$ carrier were independent factors; change in the number of silent brain infarctions was an independent factor for changes in global brain atrophy. A significant association of changes in eGFR and albuminuria with hippocampal atrophy remained after adjusting for confounders including SVD. Both types of brain atrophy at baseline were significantly correlated with cognitive impairment at baseline and especially associated with changes in delayed word recall during the follow-up after adjusting for confounders. Conclusion: Changes in eGFR and albuminuria during follow-up were independent risk factors for hippocampal atrophy, which was associated with decline in delayed word recall, suggesting that management of chronic kidney disease may prevent the progression of hippocampal atrophy. 


\section{Introduction}

The number of people with dementia is steadily increasing worldwide. It is estimated that around 4.6 million people in Japan have dementia, and it has become a serious medical and social problem. The number of older people with diabetes is also increasing as a result of an increased average life expectancy and changes in lifestyle. In previous large-scale epidemiological studies, it was reported that the incidence of dementia in diabetic patients is about twofold higher than in nondiabetic people $[1,2]$. Accordingly, an increase in the number of people with impaired glucose metabolism may have a great impact in increasing dementia prevalence. Although many mechanisms have been considered for an association between diabetes and cognitive dysfunction [3, 4], this has not yet been fully clarified. Therefore, there is an immediate need to clarify mechanisms and develop new treatments for dementia. Early diagnosis of cognitive impairment and future prediction of cognitive decline will be useful in preventing dementia progression and its associated problems.

It has been reported that atrophy of the brain, especially the medial temporal lobe, is observed at a relatively early stage of Alzheimer's disease [5]. In addition, brain atrophy in people with diabetes has been reported to be more severe as compared with nondiabetic people $[6,7]$. Magnetic resonance imaging (MRI) is in now in widespread use, and many trials have been conducted on using it to estimate brain atrophy. With the method of evaluation using voxel-based morphometry (VBM), brain atrophy can be easily semi-quantified, and Hirata et al. [8] developed a computer-assisted analysis using VBM for diagnosing Alzheimer's disease at an early stage. Free software for this procedure, called VSRAD (voxel-based specific regional analysis system for Alzheimer's disease), has made it possible to estimate hippocampal atrophy easily and speedily. It has been reported that hippocampal atrophy as estimated by VSRAD was stronger in diabetic patients as compared with nondiabetic people, and the atrophy was associated with impaired cognitive function $[9,10]$, in agreement with our recent cross-sectional study [11].

Therefore, we performed a 3-year longitudinal observation on brain atrophy as assessed with VSRAD in well-controlled elderly outpatients with type 2 diabetes in the present study. We investigated what factors were associated with progression of brain atrophy and whether brain atrophy at baseline predicted cognitive decline after 3 years.

\section{Subjects and Methods}

We performed a longitudinal observation study on cognitive impairment in elderly patients with type 2 diabetes aged more than 65 years since 2006 as previously reported [12, 13]. The present study was conducted on 74 diabetic patients, adding 13 patients newly recruited in 2009. Eight patients - 5 were institutionalized or moved, 1 died, and 2 declined participation - dropped out between 2009 and 2012. Finally, cognitive function tests were performed on 66 patients (mean $78.0 \pm 5.6$ years, 24 males and 42 females), 5 of whom did not receive MRI examinations due to pacemakers or declining them. Patients with severe cognitive impairment [Mini-Mental State Examination (MMSE) score $<19$ points] and with renal insufficiency (serum creatinine $>2.0 \mathrm{mg} / \mathrm{dl}$ ) were also excluded in 2009 .

\section{Cognitive Function Tests}

We selected the following standardized psychological tests for measurement of each function as reported previously $[12,13]$. The MMSE was used to assess orientation, registration, attention, calculation, language, and recall with a score range from 0 to 30 [14]. We performed word recall, a subtest of the Alzheimer's Disease Assessment Scale with a score 
ranging from 0 to 10 to assess verbal memory [15]. Immediately after the word list of 10 common nouns had been read, the respondents were asked to repeat as many words as they could recall in any order (immediate word recall). After approximately $30 \mathrm{~min}$ of interference tasks, the respondents were asked to recall the 10-word list again (delayed word recall). Complex psychomotor skills were evaluated by the Digit Symbol Substitution (DSS) test, a subtest of the Wechsler Adult Intelligence Scale-Revised with a score ranging from 0 to 93 [16]. We used the modified Stroop Color Word (Stroop) test consisting of 2 successive tasks in order to assess attention/executive function, in which the seconds to completion are recorded and the difference between the time required to read the word card and that required to read the dot card is calculated. A wider time difference means lower cognitive performance [17]. We calculated z-scores [(individual test score) - (mean test score)/ (standard deviation, SD)] for the neuropsychological tests except the MMSE. All neuropsychological tests were administered by a well-trained psychological tester. Depression was assessed by the Geriatric Depression Scale-15 (GDS-15), which is a global test of depression with scores ranging from 0 to 15 [18].

\section{Evaluation of Cerebral Small Vessel Disease}

MRI was performed on a 1.5-tesla Signa Horizon system (GE Medical Systems, Milwaukee, Wis., USA). The imaging protocol consisted of T1-weighted spin echo [inversion recovery; repetition time/echo time $(\mathrm{TR} / \mathrm{TE})=2,380 / 27.4 \mathrm{~ms}$, matrix $320 \times 224]$, T2-weighted fast spin echo $(\mathrm{TR} / \mathrm{TE}=4,017 / 103 \mathrm{~ms}$, matrix $156 \times 256)$, and fluid attenuated inversion recovery (FLAIR; TR/TE $=8,002 / 146 \mathrm{~ms}$, matrix $256 \times 192$ ) sequences in the axial plane with a slice thickness of $5 \mathrm{~mm}$ and a 2-mm interslice gap. We defined silent brain infarction (SBI) as focal hyperintense areas $>3 \mathrm{~mm}$ in diameter detected on T2-weighted images, hypointense areas on T1-weighted images, and areas of higher intensity than cerebrospinal fluid in FLAIR images. Lesions $<3 \mathrm{~mm}$ in diameter or with a signal intensity similar to cerebrospinal fluid in FLAIR images were excluded because of the high possibility that they were enlarged perivascular spaces, even if they exhibited hyperintensity on T2-weighted images and hypointensity on T1-weighted images. When the row number of SBI was $\geq 6$, it was counted as 6 for analytical purposes. Baseline white matter lesions (WMLs) were stratified for periventricular and subcortical areas separately. The periventricular WMLs (PWMLs) or subcortical WMLs (SWMLs) were rated according to the Fazekas scale (PWMLs: grade 0, no lesion; grade 1, caps or pencil-thin lining; grade 2 , smooth halo; grade 3 , large confluence extending into the deep white matter; SWMLs: grade 0 , no lesion; grade 1, punctate; grade 2, early confluent; grade 3; confluent) [19].

\section{Automatic VBM of Structural MRI (VSRAD)}

Hirata et al. [8] developed a computer-assisted analysis using VBM for diagnosing Alzheimer's disease at an early stage, and the free software, VSRAD, is widely available in Japan. Recently, VSRAD was improved by changing to statistical parametric mapping (SPM) 8 from SPM 2, and the use of Diffeomorphic Anatomical Registration through Exponentiated Lie Algebra (DARTEL) for standardization greatly enhanced diagnostic accuracy for Alzheimer's disease and estimation of global brain atrophy [20]. The analytical results automatically calculated by VSRAD are obtained through a comparison of the severity of gray matter atrophy in a volume of interest (VOI) at the bilateral head to tail of the hippocampus, the entorhinal cortex, and amygdala regions and global brain with its original normal database template. The normal database contains data from 80 healthy volunteers (mean age $70.4 \pm$ 7.8 years) with no memory impairment or cognitive disorders, and they are used as controls in VSRAD [20]. The z-score maps for gray matter anatomical standardization and voxel normalization to global mean intensities (global normalization) were obtained using values 
Fig. 1. Changes in severity of gray matter atrophy in the VOI (HAI) during the 3-year follow-up in the case of a 78-year-old female. a MRI. Progression of medial temporal lobe atrophy was observed on FLAIR MRI during the 3 years. b VSRAD. Colored areas (colors refer to the online version only) with z-scores of $>2$ are overlaid as significantly atrophied regions on the standardized MRI template. The target VOI is surrounded by purple lines (white arrows), and red areas indicate higher gray matter atrophy levels as compared with the control (normal database). The HAI (z-score) increased from 1.57 to 3.20 , and the MMSE dropped from 23 to 17 after 3 years.

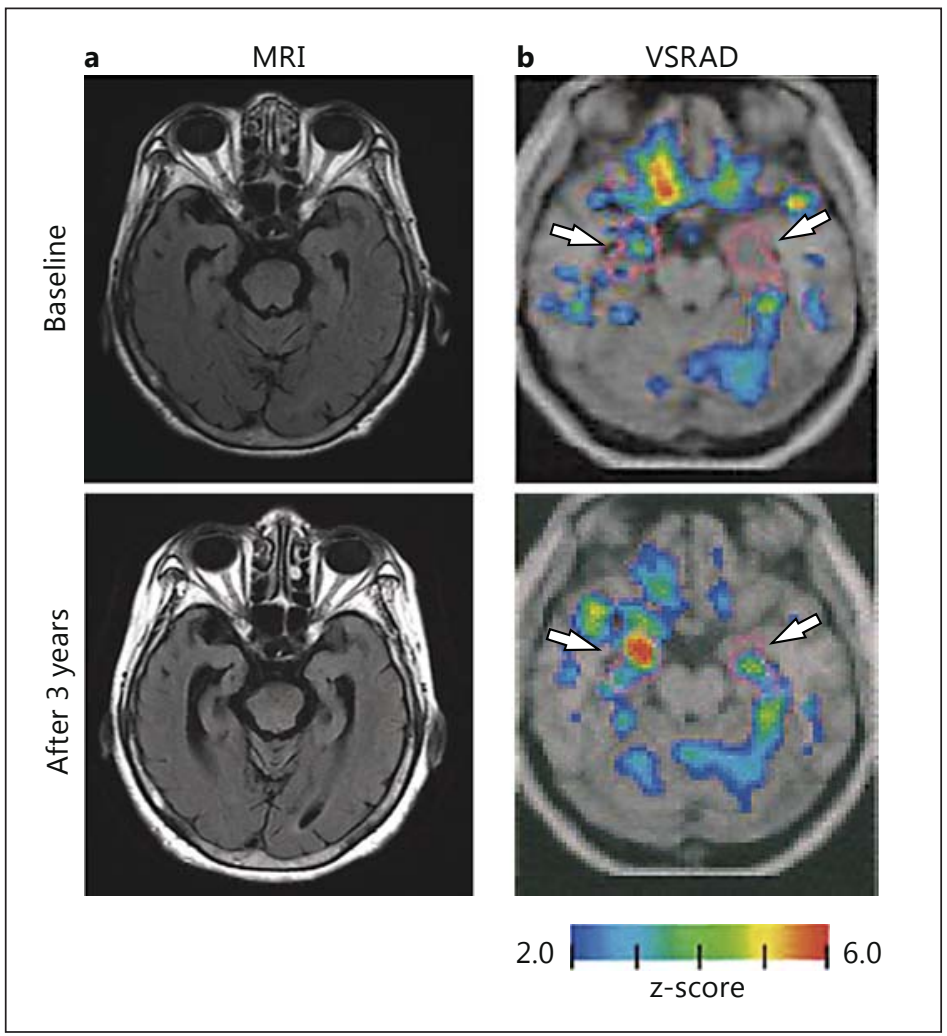

calculated by the following equation: $z$-score $=[($ control mean $)-($ individual value $)] /($ control SD). The z-score maps are displayed by overlay on tomographic sections and surface rendering of the standardized brain. The severity of atrophy in a target VOI was defined as the mean value of z-scores $>0$ in the VOI, and it was expressed as the hippocampal atrophy index (HAI). Figure 1 shows a case of progression in medial temporal lobe atrophy and HAI. In addition, the extent of gray matter atrophy was calculated as the percentage of coordinates with a $\mathrm{z}$-score exceeding the threshold value of 2 in the whole brain [whole-brain atrophy index (WBAI) in \%).

\section{Evaluation of Patients}

Fasting blood samples were separated and analyzed. Low-density lipoprotein (LDL)cholesterol, high-density lipoprotein (HDL)-cholesterol, triglycerides, and fasting blood glucose were measured using an autoanalyzer and routine enzymatic techniques. Hemoglobin $\mathrm{A}_{1 \mathrm{c}}\left(\mathrm{HbA}_{1 \mathrm{c}}\right)$ was measured using high-performance liquid chromatography (HLC723G7; Tosoh, Tokyo, Japan), and $\mathrm{HbA}_{1 \mathrm{c}}$ was estimated as a National Glycohemoglobin Standardization Program (NGSP) value (in \%), calculated by the formula $\mathrm{HbA}_{1 \mathrm{c}}(\%)=\left[\mathrm{HbA}_{1 \mathrm{c}}\right.$ Japan Diabetes Society (JDS)] (\%) × $1.02+0.25$ [21]. Plasma insulin was measured by chemiluminescence enzyme immunoassay. Inflammatory markers were assayed using the quantitative sandwich enzyme technique of the enzyme-linked immunosorbent assay QuantiGlo kit (R\&D Systems, Minneapolis, Minn., USA) for tumor necrosis factor- $\alpha$ (TNF- $\alpha$ ) and interleukin-6, and an immunosorbent assay kit (R\&D Systems, Abingdon, UK) for soluble intercellular adhesion molecule 1 (sICAM-1) as reported previously [22]. High-sensitivity C-reactive protein was assayed using a monoclonal antibody coated onto polystyrene particles and fixed-timed kinetic nephelometric measurements (BN II; Dade Behring, Marberg, Germany). 
Apolipoprotein (Apo) E genotype was determined for each participant by the Invader assay [23]. The Apo E genotype was divided into 2 groups, one with $\varepsilon 4(3 / 4$ and $4 / 4)$ and one without $\varepsilon 4(3 / 3$ and $3 / 2)$.

The estimated glomerular filtration rate (eGFR) was calculated from the following 3-variable Japanese equation: GFR $\left(\mathrm{ml} / \mathrm{min} / 1.73 \mathrm{~m}^{2}\right)=194 \times$ serum $^{2}$ creatinine ${ }^{-1.094} \times$ age $^{-0.287} \times 0.739$ (if female) [24]. A low eGFR was defined as $<60 \mathrm{ml} / \mathrm{min} / 1.73 \mathrm{~m}^{2}$. Albuminuria was defined as albumin-to-creatinine ratios (ACRs), which were calculated from the measurement of albumin (in $\mathrm{mg}$ ) and creatinine (in g) in spot urine, as follows: normoalbuminuria, $<30 \mathrm{mg} / \mathrm{g}$ creatinine; microalbuminuria, 30-299 mg/g creatinine, and macroalbuminuria, $\geq 300 \mathrm{mg} / \mathrm{g}$ creatinine. Also, owing to the small number of participants with macroalbuminuria, micro- and macroalbuminuria were considered together as albuminuria. Diabetic retinopathy was classified as normal, simple and preproliferative, and proliferative retinopathy by ophthalmologists.

This study was approved by the Ethics Committee of Chubu Rosai Hospital. After informed consent was obtained from each of the participants, the study was performed in accordance with the principles of the Declaration of Helsinki.

\section{Statistical Analysis}

Results are expressed as means \pm SD. Statistical analysis was performed by the paired $t$ test on comparisons between baseline and after the 3-year follow-up. Comparisons for factors not forming normal distributions were performed by the Wilcoxon signed-rank test, a nonparametric statistical method, and results are expressed as medians (interquartile range, $25-75 \%)$. The $\chi^{2}$ test was also conducted for independence. Comparisons between the groups were carried out using ANOVA followed by Bonferroni's test. We carried out multiple linear regression or multiple logistic regression where appropriate, and variables with $p<0.05$ in univariate analysis were entered for multiple regression analysis. Also, if 2 variables had collinearity, one of them was not entered. Statistical analyses were carried out using SPSS software 21.0 (SPSS, Chicago, Ill., USA), and $\mathrm{p}<0.05$ was considered to be statistically significant.

\section{Results}

\section{Clinical and Biochemical Characteristics at Baseline and after 3 Years of Follow-Up in} Elderly Diabetic Patients

Diabetic patients were well controlled during the 3 years with an average $\mathrm{HbA}_{1 \mathrm{c}}$ of 6.95 $\pm 0.62 \%$ (table 1). The average blood pressure during follow-up was $136.3 \pm 10.6 / 72.9 \pm 6.5$ $\mathrm{mm}$ Hg. After 3 years, HDL-cholesterol, diastolic blood pressure, and eGFR values were decreased, and TNF- $\alpha$, uric acid, and sICAM-1 levels were increased significantly, as compared with baseline values. Also, the rates of patients with a low eGFR $\left(<60 \mathrm{ml} / \mathrm{min} / 1.73 \mathrm{~m}^{2}\right)$ and albuminuria had significantly increased after 3 years ( $p=0.01$ and $p=0.03$, respectively). As for cognitive function, scores for the MMSE, immediate word recall, and DSS test had declined after 3 years (MMSE score $25.4 \pm 3.1$ at baseline vs. $24.8 \pm 3.7$ at 3 years of follow-up, $p=$ 0.081 ; immediate word recall score $6.64 \pm 1.31$ vs. $6.25 \pm 1.67, \mathrm{p}=0.021$; delayed word recall score $6.47 \pm 2.141$ vs. $6.24 \pm 2.74, \mathrm{p}=0.313$; DSS test score $34.1 \pm 10.6$ vs. $31.3 \pm 11.7, \mathrm{p}<$ 0.001 ; Stroop test score $20.3 \pm 13.9$ vs. $19.0 \pm 11.5$, p = 0.416).

During the 3-year follow-up, SBI progression was noted in 5 patients $(8.2 \%)$, and progression of PWMLs and SWMLs was observed in 6 (9.8\%) and 3 patients (4.9\%), respectively. As for brain atrophy, the HAI increased from $1.50 \pm 0.76$ to $1.75 \pm 0.94(0.083$ per year; $\mathrm{p}=0.001)$, and the WBAI increased from $8.65 \pm 2.62$ to $9.78 \pm 2.93 \%(\mathrm{p}<0.001)$. 
Table 1. Clinical and biochemical characteristics at baseline and after 3 years of follow-up in elderly diabetic patients

\begin{tabular}{|c|c|c|c|}
\hline & Baseline & After 3 years & $\mathrm{p}$ value \\
\hline Duration of diabetes, years & $21.8 \pm 8.8$ & - & - \\
\hline Education, years & $9.47 \pm 1.80$ & - & - \\
\hline ApoE $\varepsilon 4$ (yes/no) & $10 / 56(15.2 \%)$ & - & - \\
\hline Smoking (never/ex-/current) & $43 / 14 / 9$ & $43 / 18 / 5$ & - \\
\hline Treatment (diet/OHAs/insulin) & $13 / 41 / 12$ & $13 / 41 / 12$ & - \\
\hline Nephropathy (normo-/micro-/macroalbuminuria) & $48 / 11 / 7$ & $37 / 22 / 7$ & - \\
\hline Retinopathy (no/simple/proliferative) & $44 / 10 / 12$ & $46 / 9 / 11$ & - \\
\hline Body mass index & $23.1 \pm 3.8$ & $23.2 \pm 4.1$ & 0.77 \\
\hline Waist circumference, $\mathrm{cm}$ & $84.6 \pm 11.0$ & $85.2 \pm 11.4$ & 0.54 \\
\hline Systolic blood pressure, $\mathrm{mm} \mathrm{Hg}$ & $137.5 \pm 14.9$ & $133.6 \pm 18.7$ & 0.15 \\
\hline Diastolic blood pressure, $\mathrm{mm} \mathrm{Hg}$ & $74.3 \pm 8.3$ & $70.8 \pm 11.4$ & 0.02 \\
\hline RAS blocker use (yes/no) & $42 / 24(63.6 \%)$ & $45 / 21(68.2 \%)$ & 0.45 \\
\hline HDL-cholesterol, mg/dl & $57.7 \pm 14.9$ & $54.0 \pm 15.6$ & $<0.01$ \\
\hline Triglycerides, mg/dl & $100.0(73.0-140.0)$ & $104.0(73.0-141.0)$ & 0.69 \\
\hline LDL-cholesterol, mg/dl & $108.6 \pm 21.4$ & $103.7 \pm 27.5$ & 0.13 \\
\hline Statin use (yes/no) & $39 / 27$ (59.1\%) & $38 / 28(57.6 \%)$ & 1.00 \\
\hline Fasting blood glucose, mg/dl & $127.6 \pm 32.3$ & $132.7 \pm 31.0$ & 0.36 \\
\hline Fasting insulin ${ }^{\mathrm{a}}, \mu \mathrm{U} / \mathrm{ml}$ & $5.10(4.00-8.08)$ & $4.90(3.20-8.00)$ & 0.47 \\
\hline $\mathrm{HbA}_{1 \mathrm{c}}, \%$ & $7.04 \pm 0.77$ & $6.92 \pm 0.69$ & 0.13 \\
\hline Serum creatinine, $\mathrm{mg} / \mathrm{dl}$ & $0.78 \pm 0.23$ & $0.86 \pm 0.26$ & $<0.001$ \\
\hline $\mathrm{eGFR}, \mathrm{ml} / \mathrm{min} / 1.73 \mathrm{~m}^{2}$ & $65.4 \pm 15.6$ & $59.4 \pm 17.8$ & $<0.001$ \\
\hline eGFR $<60 \mathrm{ml} / \mathrm{min} / 1.73 \mathrm{~m}^{2}$ (yes/no) & $23 / 43(34.8 \%)$ & $36 / 30(54.5 \%)$ & 0.01 \\
\hline Urinary ACR, mg/g creatinine & $26.0(15.0-49.0)$ & $23.0(15.0-49.0)$ & 0.48 \\
\hline Albumin, g/dl & $4.24 \pm 0.33$ & $4.28 \pm 0.53$ & 0.51 \\
\hline Uric acid, mg/dl & $5.23 \pm 1.28$ & $5.64 \pm 1.50$ & $<0.01$ \\
\hline hs-CRP, mg/l & $0.30(0.20-0.80)$ & $0.44(0.25-1.53)$ & 0.11 \\
\hline sICAM-1, $\mu \mathrm{g} / \mathrm{l}$ & $203.5 \pm 65.1$ & $218.5 \pm 83.3$ & $<0.01$ \\
\hline TNF- $\alpha, \mathrm{pg} / \mathrm{ml}$ & $1.65(0.94-2.30)$ & $1.98(1.38-2.57)$ & 0.02 \\
\hline IL-6, pg/ml & $1.18(0.78-1.84)$ & $1.29(0.83-2.19)$ & 0.13 \\
\hline
\end{tabular}

Data are expressed as means \pm SD or medians (interquartile range, 25-75\%) unless specified otherwise. OHAs = Oral hypoglycemic agents; RAS blocker = renin angiotensin system blocker; hs-CRP = high-sensitivity C-reactive protein; IL-6 = interleukin-6. ${ }^{\text {a }}$ Insulin users are excluded.

Factors Associated with Brain Atrophy, HAI, and WBAI

HAI was significantly correlated with GDS score, age, a low eGFR, and the number of SBI at baseline, and multivariate analysis showed that GDS score (standardized $\beta=0.225$, SE $=$ $0.028, p=0.078)$ and a low eGFR $(\beta=0.203, \mathrm{SE}=0.211, \mathrm{p}=0.098)$ had a tendency to be associated with HAI $\left(r^{2}=0.229, p=0.003\right)$. In contrast, WBAI was significantly correlated with age, sex, GDS score, the number of SBIs, PWML grade, SWML grade, eGFR, and levels of serum albumin, sICAM-1, log TNF- $\alpha$, and LDL-cholesterol, and multivariate analysis showed that age $(\beta=0.341, \mathrm{SE}=0.062, \mathrm{p}=0.013)$, GDS score $(\beta=0.247, \mathrm{SE}=0.072, \mathrm{p}=0.023)$, and SWML grade $(\beta=0.349, \mathrm{SE}=0.452, \mathrm{p}=0.016)$ were associated with WBAI $\left(\mathrm{r}^{2}=0.583, \mathrm{p}<0.001\right)$.

Factors associated with changes in HAI and WBAI during the 3-year follow-up are shown in table 2. Changes in HAI were significantly correlated with changes in eGFR, age, diastolic blood pressure at baseline, changes in HDL-cholesterol, ApoE $\varepsilon 4$ carrier, and albuminuria during follow-up in univariate analysis, and multivariate analysis showed that changes in eGFR, ApoE $\varepsilon 4$ carrier, and albuminuria during follow-up were independent factors for 
Table 2. Factors associated with changes in $\mathrm{HAI}(\triangle \mathrm{HAI})$ and WBAI $(\Delta \mathrm{WBAI})$ during the 3-year follow-up

\begin{tabular}{|c|c|c|c|c|}
\hline & \multicolumn{2}{|c|}{ Univariate analysis } & \multicolumn{2}{|l|}{ Multivariate analysis } \\
\hline & $\begin{array}{l}\text { correlation } \\
\text { coefficient }\end{array}$ & $\mathrm{p}$ value & $\begin{array}{l}\text { standardized } \beta \\
\text { coefficient }(\mathrm{SE})\end{array}$ & $\mathrm{p}$ value \\
\hline$\Delta \mathrm{HAI}$ & & & $r^{2}=0.388, p<0.001$ & \\
\hline$\Delta \mathrm{eGFR}\left(\mathrm{ml} / \mathrm{min} / 1.73 \mathrm{~m}^{2}\right)$ & -0.396 & 0.002 & $-0.282(0.007)$ & 0.022 \\
\hline Age (years) & 0.377 & 0.003 & $0.213(0.012)$ & 0.074 \\
\hline DBP at base (mm Hg) & -0.297 & 0.020 & $-0.096(0.007)$ & 0.425 \\
\hline$\Delta$ HDL-cholesterol (mg/dl) & -0.288 & 0.025 & $-0.151(0.007)$ & 0.461 \\
\hline ApoE $\varepsilon 4$ carrier & 0.277 & 0.031 & $0.285(0.178)$ & 0.013 \\
\hline Albuminuria during follow-up & 0.267 & 0.037 & $0.232(0.120)$ & 0.038 \\
\hline$\Delta \mathrm{WBAI}$ & & & $r^{2}=0.295, p=0.008$ & \\
\hline$\triangle \mathrm{PWML}$ grade & 0.306 & 0.016 & $0.196(0.728)$ & 0.123 \\
\hline Waist circumference at base $(\mathrm{cm})$ & 0.299 & 0.020 & $0.214(0.022)$ & 0.145 \\
\hline Duration of diabetes (years) & 0.276 & 0.031 & $0.173(0.027)$ & 0.223 \\
\hline Treatment modality & 0.276 & 0.031 & $0.063(0.445)$ & 0.694 \\
\hline Retinopathy & 0.272 & 0.034 & $0.082(0.307)$ & 0.578 \\
\hline $\log$ CRP at base & 0.253 & 0.049 & $0.036(0.403)$ & 0.797 \\
\hline$\Delta \mathrm{SBI}$ (number of infarcts) & 0.253 & 0.049 & $0.266(0.697)$ & 0.031 \\
\hline
\end{tabular}

Variables with $\mathrm{p}<0.05$ in univariate analysis were entered into the multiple regression analysis. Retinopathy: $0=$ none; 1 = simple; 2 = proliferative. Treatment modality: $0=$ diet; 1 = oral hypoglycemic agents; 2 = insulin. DBP = Diastolic blood pressure; CRP = C-reactive protein.

changes in HAI. In contrast, although changes in WBAI were significantly correlated with the factors listed in table 2, only change in the number of SBIs was an independent factor in multivariate analysis.

With regard to an association of brain atrophy with renal impairment, changes in eGFR were significantly correlated with changes in HAI (fig. 2a), but not in WBAI, and the correlation remained significant after adjusting for covariates such as age, sex, ApoE $\varepsilon 4$ carrier, HAI and eGFR at baseline, and albuminuria $(\mathrm{p}=0.031)$. In contrast, although changes in ACR were not correlated with brain atrophy (data not shown), a significant increase in HAI was observed in patients with albuminuria as compared with patients without albuminuria during the follow-up $(0.423 \pm 0.664$ vs. $0.129 \pm 0.410, p=0.037)$, and the significance remained after adjusting for age, sex, ApoE $\varepsilon 4$ carrier, changes in eGFR, and HAI at baseline (odds ratio 4.81, $95 \%$ CI 1.26-18.37, $\mathrm{p}=0.022$ ). Also, the HAI showed a tendency to gradually increase in patients with newly detected albuminuria $(0.343 \pm 0.375)$ and persistent albuminuria $(0.502$ \pm 0.874 ) as compared with those without albuminuria ( $p=0.087$ ) (fig. $2 b$ ).

\section{Association between Brain Atrophy and Cognitive Function Tests}

At baseline, HAI was correlated with scores on the MMSE $(r=-0.282, p=0.015)$, immediate word recall $(r=-0.203, p=0.083)$, delayed word recall $(r=-0.276, p=0.017)$, and the DSS test $(r=-0.345, p=0.003)$, and WBAI was correlated with scores on the MMSE $(r=$ $-0.390, \mathrm{p}=0.001)$, immediate word recall $(\mathrm{r}=-0.299, \mathrm{p}=0.010)$, delayed word recall $(\mathrm{r}=$ $-0.281, \mathrm{p}=0.015)$, the DSS test $(\mathrm{r}=-0.419, \mathrm{p}<0.001)$, and the Stroop test $(\mathrm{r}=0.244, \mathrm{p}=$ $0.036)$.

An association between brain atrophy at baseline and changes in cognitive function tests during the 3-year follow-up is indicated in table 3. Baseline HAI was significantly correlated with changes in delayed word recall (fig. 3a) and Stroop test scores, and these significant 


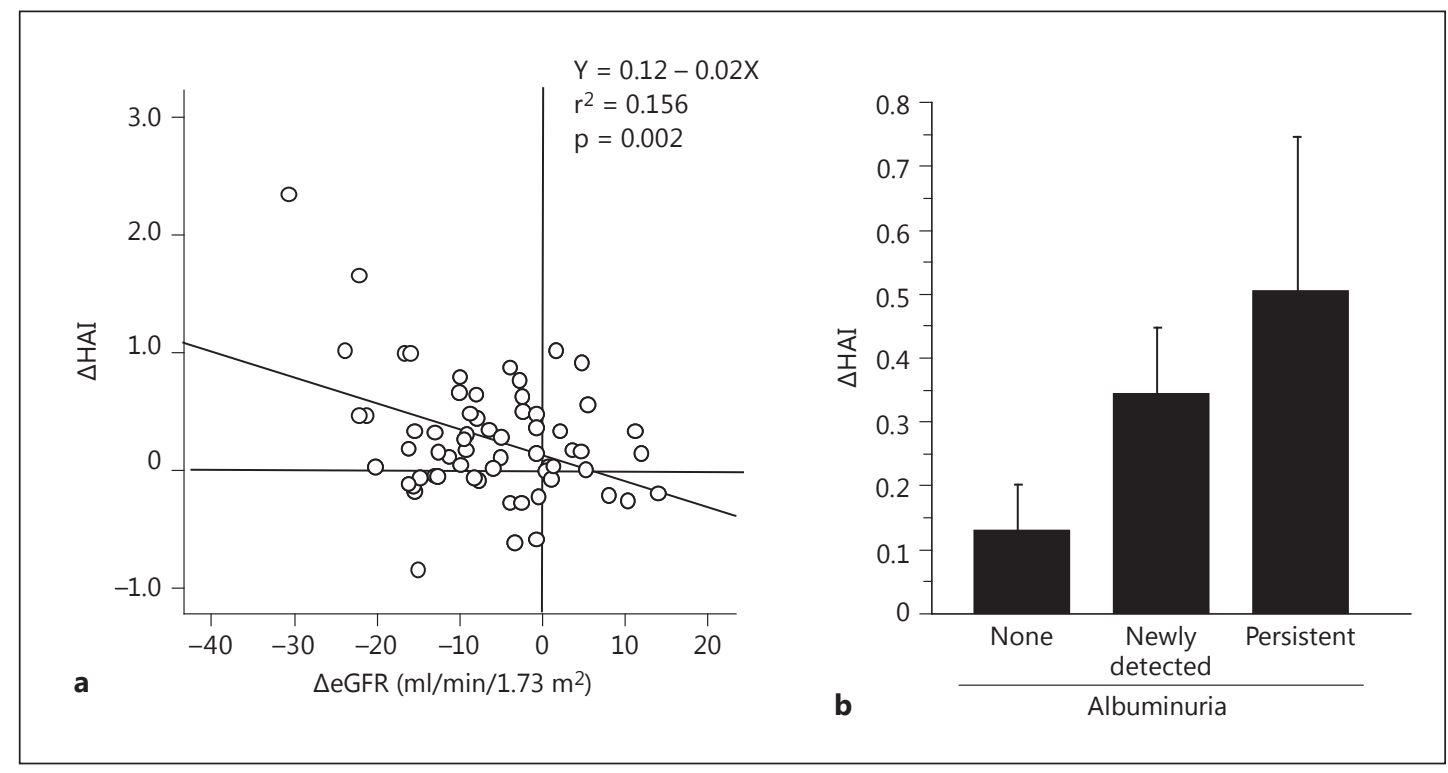

Fig. 2. Association between changes in $H A I(\triangle H A I)$ and changes in eGFR $(\Delta e G F R)$ and albuminuria during the 3 -year follow-up. Albuminuria was divided into 3 categories during the follow-up period: normoalbuminuria or regression to normoalbuminuria from albuminuria ('none'), progression to albuminuria from normoalbuminuria ('newly detected'), and persistent albuminuria during the follow-up period ('persistent'). Comparisons between the 3 groups were conducted by ANOVA (mean $\pm \mathrm{SE}, \mathrm{p}=0.087$ ).

Table 3. Associations between HAI and WBAI at baseline and changes in cognitive function domains during the 3-year follow-up in elderly diabetic patients

\begin{tabular}{|c|c|c|c|c|c|}
\hline & \multirow{2}{*}{$\begin{array}{l}\triangle \mathrm{MMSE} \\
\text { score }\end{array}$} & \multicolumn{2}{|l|}{$\Delta$ word recall score } & \multirow{2}{*}{$\begin{array}{l}\Delta \text { DSS test } \\
\text { score }\end{array}$} & \multirow{2}{*}{$\begin{array}{l}\Delta \text { Stroop test } \\
\text { score }\end{array}$} \\
\hline & & $\Delta$ immediate & $\Delta$ delayed & & \\
\hline \multicolumn{6}{|c|}{ HAI at baseline } \\
\hline Model 1 & $-0.184(0.113)$ & $-0.161(0.129)$ & $-0.340(0.095)^{* *}$ & $-0.080(0.065)$ & $0.251(0.155)^{*}$ \\
\hline Model 2 & $-0.228(0.116)^{\#}$ & $-0.135(0.132)$ & $-0.348(0.104)^{* *}$ & $-0.010(0.074)$ & $0.233(0.126)^{*}$ \\
\hline Model 3 & $-0.290(0.143)^{\#}$ & $-0.155(0.155)$ & $-0.298(0.106)^{*}$ & $-0.089(0.086)$ & $0.295(0.152)^{*}$ \\
\hline \multicolumn{6}{|c|}{ WBAI at baseline } \\
\hline Model 1 & $-0.035(0.037)$ & $-0.352(0.040)^{* * *}$ & $-0.429(0.030)^{* * * *}$ & $-0.262(0.022)^{*}$ & $0.032(0.054)$ \\
\hline Model 2 & $-0.048(0.047)$ & $-0.379(0.047)^{* *}$ & $-0.496(0.037)^{* * *}$ & $-0.234(0.028)$ & $0.074(0.055)$ \\
\hline Model 3 & $-0.002(0.052)$ & $-0.458(0.134)^{* * *}$ & $-0.471(0.034)^{* * *}$ & $-0.130(0.029)$ & $0.062(0.058)$ \\
\hline
\end{tabular}

In the multivariate analysis, changes in cognitive function domains were used as the dependent variable. Values are standardized $\beta$ and coefficients (SE). ${ }^{*} \mathrm{p}<0.1,{ }^{*} \mathrm{p}<0.05,{ }^{* *} \mathrm{p}<0.01,{ }^{* * *} \mathrm{p}<0.005,{ }^{* * * *} \mathrm{p}<0.001$. Model 1: no adjustment. Model 2: adjustment for age, sex, years of education, and each cognitive function domain at baseline. Model 3: adjustment for duration of diabetes, ApoE $\varepsilon 4$ carrier, the mean of systolic blood pressure, diastolic blood pressure, and $\mathrm{HbA}_{1 \mathrm{c}}$ during follow-up, eGFR $<60 \mathrm{ml} / \mathrm{min} / 1.73 \mathrm{~m}^{2}$, albuminuria, treatment modality $(0=$ diet, $1=$ oral hypoglycemic agents, $2=$ insulin $)$, and current smoking $(0=$ no, $1=$ yes $)$ in addition to the factors in model 2. 
Kawamura et al.: Factors Associated with Changes in Brain Atrophy during a

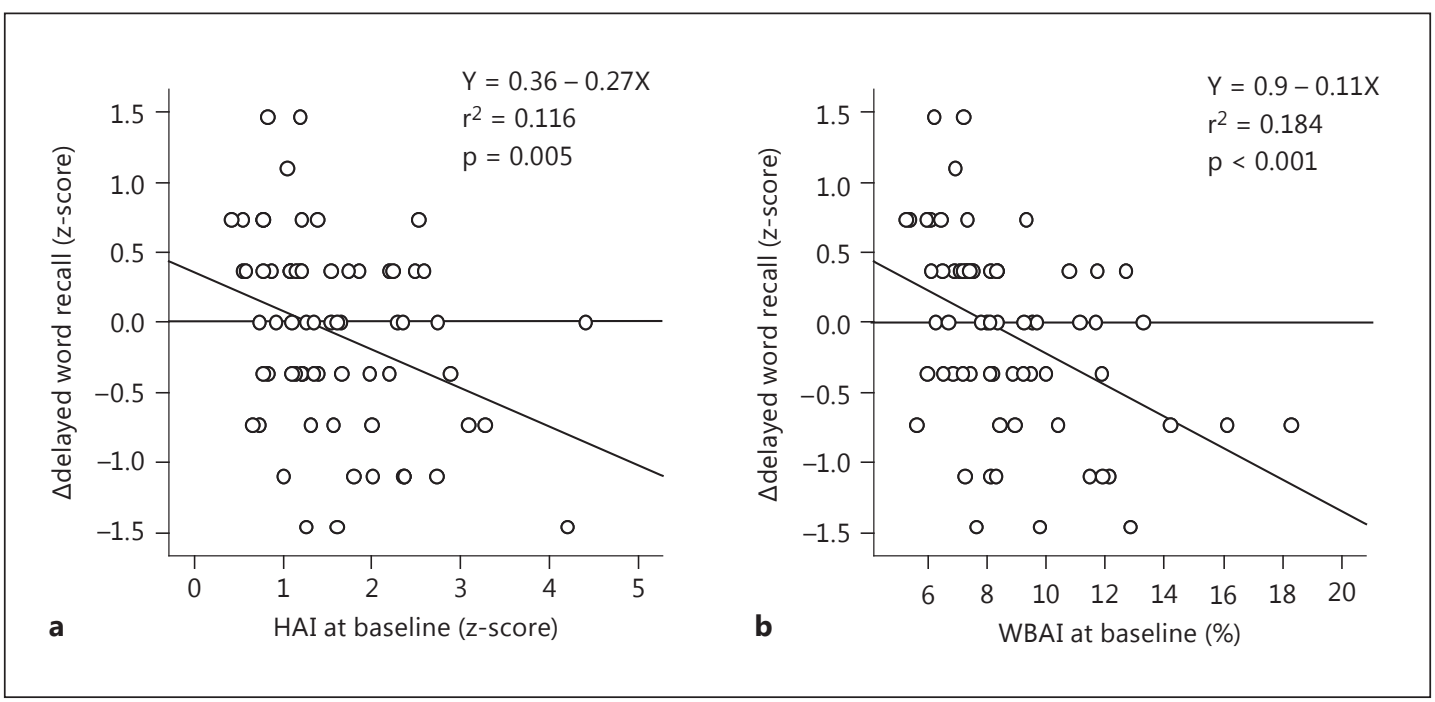

Fig. 3. Association of changes $(\Delta)$ in delayed word recall with HAI and WBAI at baseline.

associations remained after adjusting for covariates. In contrast, baseline WBAI was significantly correlated with changes in scores of immediate and delayed word recall after adjusting for covariates (fig. 3b). Also, such significant associations still remained after adding the number of infarcts and the grade of WMLs at baseline, or their changes during the follow-up, to the covariates (data not shown).

With regard to associations between changes in brain atrophy and cognitive function tests during the 3-year follow-up, changes in HAI were only correlated with changes in DSS test scores, and the relationship remained significant after adjusting for age, sex, years of education, and baseline scores $(\beta=-0.293, \mathrm{SE}=0.145, \mathrm{p}=0.019)$. In contrast, no significant relationship was observed between changes in WBAI and cognitive function tests.

\section{Discussion}

A large number of studies have shown that a smaller brain volume due to atrophy, especially hippocampal atrophy, on MRI was noted in subjects with Alzheimer's disease or mild cognitive impairment as compared with those without, and such atrophy was associated with cognitive decline and incident dementia [5]. It is well known that impaired cognitive function is more prevalent in diabetic patients as compared with nondiabetic subjects, from large epidemiological studies [1, 2], and it has also been reported that brain atrophy was greater in diabetic patients than in nondiabetic subjects [6, 7]. Although it has often been reported that factors such as blood glucose, duration of diabetes, insulin resistance, involvement of hypertension, and cerebrovascular disease are associated with brain atrophy in diabetic patients $[6,7,25,26]$, the mechanisms by which greater brain atrophy occurs in diabetic patients have not been fully resolved.

Our present 3-year observation showed that progression of hippocampal atrophy was independently associated with ApoE $\varepsilon 4$ genotype, changes in eGFR, and albuminuria. In contrast, progression of global atrophy was associated with only changes in lacunar infarcts. So far, a positive relationship between brain volumes and glucose levels has often been reported $[27,28]$, and the ACCORD MIND study showed that less brain atrophy was found in 
strict control groups as compared with standard control groups [29]. However, most studies in a recent systematic review showed that $\mathrm{HbA}_{1 \mathrm{c}}$ levels were not associated with the presence of brain atrophy and changes in brain volumes in type 2 diabetes [30], in accordance with the present study.

Thus, although the present study revealed no significant independent associations of diabetes characteristics, such as glucose metabolism and insulin resistance, with changes in brain atrophy, further large-scale studies will be needed to clarify such associations. However, interestingly, it is noteworthy that renal impairment was independently associated with brain atrophy, especially hippocampal atrophy, in the present study. Sink et al. [31] have recently reported that urinary ACRs were significantly associated with global gray matter atrophy and eGFR was associated with WML volumes in hippocampal lesions in African Americans with type 2 diabetes. Also, Mehta et al. [32] have reported that subclinical albuminuria was associated with gray matter atrophy in the global brain in type 2 diabetes. In addition, associations of GFR and albuminuria with brain atrophy have been reported in nondiabetic subjects $[33,34]$. However, it has also been observed that microvascular complications such as albuminuria were not associated with brain volumes or volume changes [35]. In this regard, our previous cross-sectional study showed that global atrophy was associated with both eGFR $<60 \mathrm{ml} / \mathrm{min} / 1.73 \mathrm{~m}^{2}$ and albuminuria, while hippocampal atrophy was associated with albuminuria [11]. In the present observation, changes in albuminuria and eGFR were associated with changes in hippocampal atrophy, but not global atrophy. To our knowledge, there have been few studies on a relationship of renal impairment with hippocampal atrophy. The reason why changes in renal impairment were associated with changes in hippocampal lesions but not those in global brain atrophy was not clear, and further studies will be needed to verify this.

In addition, although the mechanisms underlying the pathological association between renal impairment and brain atrophy remain unclear, several hypotheses might explain such an association. Generally, the brain and kidney could be considered as end organs having similar low-resistance vascular beds and endothelial structures. Endothelial dysfunction in the kidney might contribute to impaired glomerular filtration resulting from a reduction of nitric oxide synthase activity and secondary protein leakage [36], while dysfunctions in the brain might decrease the clearance of $\beta$-amyloid and lead to the accumulation of neurotoxic substances resulting from blood-brain barrier damage, leading to brain atrophy and cognitive impairment [37]. Albuminuria is thought to be a marker of endothelial dysfunction, and many studies have been conducted in regard to an association between it and cognitive impairment $[38,39]$. Our previous study showed that changes in albuminuria were correlated with decline in word recall [40], and that serum levels of ICAM-1, which reflect endothelial damage, were associated with executive function assessed with the DSS test [41].

From another viewpoint, the majority of studies have reported that the presence and progression of small vessel disease (SVD) were associated with brain atrophy in nondiabetic $[42,43]$ and diabetic subjects $[25,44]$. Although the mechanisms for an association of SVD with brain atrophy also remain unclear, one possible explanation is that the hypoxic-ischemic condition brought about by SVD damages brain tissues such as myelin and axons, leading to shrinking of the brain. However, a connection between SVD and brain atrophy has not always been established, because results have differed depending on whether the SVD consisted of lacunar infarcts or WMLs, the atrophy was global or regional, or the study design was crosssectional or longitudinal. In fact, WMLs were associated with global brain atrophy at baseline, while changes in lacunar infarcts were longitudinally associated with changes in global brain atrophy in the present study. We did not find an association of SVD with hippocampal atrophy.

Regarding an association between cognitive domains and brain atrophy, it has been reported that brain atrophy was associated with impairment of information processing 
Kawamura et al.: Factors Associated with Changes in Brain Atrophy during a

Three-Year Observation in Elderly Diabetic Patients

speed, attention, executive function, and memory [6, 7]. Our study also showed that both global and hippocampal atrophy were associated with memory, psychomotor speed, and executive function, while changes in hippocampal atrophy were only associated with changes in psychomotor speed during the follow-up. In addition, hippocampal atrophy at baseline was a predictor of decline in delayed memory and executive function, while global atrophy at baseline was a predictor of decline in both immediate and delayed memory after adjusting for confounders. Most cross-sectional studies have reported an association between brain volumes and executive functioning or processing speed [6]. In contrast, a longitudinal study by van Elderen et al. [45] reported that global atrophy at baseline was a predictor of immediate verbal memory loss, which is basically consistent with our results. Also, another longitudinal study showed that progressive medial temporal atrophy, but not baseline gray matter, predicted a significant decrease in memory function [26]. In any case, one may say that brain atrophy is a good predictor of future cognitive decline, although there only is an association for some cognitive domains but not for others.

Several limitations of this study should be taken into consideration. First, it was a smallscale hospital-based study, and treatment according to the guidelines may have influenced the results. In addition, it was unclear whether the results of the present study were peculiar to diabetes and independent of aging, since this was not investigated in nondiabetic subjects. Therefore, further large-scale studies will be needed to verify our results. Second, cognitive impairment due to vascular dementia was indistinguishable from that due to Alzheimer's disease. Third, the free VSRAD software was developed as a tool for VBM using data from a Japanese database; thus, if it is going to be used for people of other ethnic groups, it will be necessary to modify the software to suit databases for such people.

In conclusion, changes in eGFR and albuminuria were independent risk factors for hippocampal atrophy, which was associated with decline in word recall, suggesting that the management of chronic kidney disease may prevent progression of hippocampal atrophy. In addition, the usefulness of VSRAD, a simple and easy-to-use tool, in predicting the progression of cognitive decline associated with brain atrophy was demonstrated in the daily clinical setting.

\section{Acknowledgements}

This research was supported by research funds to promote hospital function from the Japan Labor Health and Welfare Organization.

\section{Disclosure Statement}

The authors have no conflicts of interest to declare.

\section{References}

1 Ott A, Stolk RP, van Harskamp F, Pois HAP, Hofman A, Breteler MMB: Diabetes mellitus and the risk of dementia. The Rotterdam Study. Neurology 1999;53:1937-1942.

-2 Gudala K, Bansal D, Schifano F, Bhansali A: Diabetes mellitus and risk of dementia: a meta-analysis of prospective observational studies. J Diabetes Investig 2013;4:640-650.

-3 Biessels GJ, Staekenborg S, Brunner E, Brayne C, Scheltens P: Risk of dementia in diabetes mellitus: a systematic review. Lancet Neurol 2006;5:69-74.

-4 Kawamura T, Umemura T, Hotta N: Cognitive impairment in diabetic patients: can diabetic control prevent cognitive decline? J Diabetes Investig 2012;3:413-423. 
5 den Heijer T, van der Lijin F, Koudstaal PJ, Hofman A, van der Lugt A, Krestin GP, Niessen WJ, Breteler MMB: A 10-year follow-up of hippocampal volume on magnetic resonance imaging in early dementia and cognitive decline. Brain 2010;133:1163-1172.

6 Biessels GJ, Reijimer YD: Brain changes underlying cognitive dysfunction in diabetes: what can we learn from MRI? Diabetes 2014;63:2244-2252.

7 Brundel M, Kappelle LJ, Biessels GJ: Brain imaging in type 2 diabetes. Eur Neuropsychopharmacol 2014;24: 1967-1981.

8 Hirata Y, Matsuda H, Nemoto K, Ohnishi T, Hirao K, Yamashita F, Asada T, Iwabuchi S, Samejima H: Voxel-based morphometry to discriminate early Alzheimer's disease from controls. Neurosci Lett 2005;382:269-274.

-9 Kamiyama K, Wada A, Sugihara M, Kurioka S, Hayashi K, Hayashi T, Yoshisako T, Yamamoto N, Tsuchie Y, Yamaguchi S, Sugimoto T, Kitagaki H: Potential hippocampal region atrophy in diabetes mellitus type 2: a voxel-based morphometry VSRAD study. Jpn J Radiol 2010;28:266-272.

10 Hayashi K, Kurioka S, Yamaguchi T, Morita M, Kanazawa I, Takase H, Wada A, Kitagaki H, Nagai A, Bokura H, Yamaguchi S, Sugimoto T: Association of cognitive dysfunction with hippocampal atrophy in elderly Japanese people with type 2 diabetes. Diabetes Res Clin Pract 2011;94:180-185.

11 Kawamura T, Umemura T, Imamine R, Umegaki H, Kawano N, Mizoguchi A, Kawai M, Minatoguchi M, Kusama M, Kouchi Y, Watarai A, Kanai A, Nakashima E, Hotta N: Factors associated with brain atrophy estimated with automatic voxel-based morphometry of structural magnetic resonance images in elderly diabetic patients: impact of albuminuria on hippocampal atrophy. J Diabetes Metab 2015;6:491.

-12 Umegaki H, Kawamura T, Mogi N, Umemura T, Kanai A, Sano T: Glucose control levels, ischaemic brain lesions, and hyperinsulinaemia were associated with cognitive dysfunction in diabetic elderly. Age Aging 2008;37: $458-461$.

-13 Imamine R, Kawamura T, Umemura T, Umegaki H, Kawano N, Hotta M, Kouchi Y, Hatsuda S, Watarai A, Kanai A, Nakashima E, Sano T, Sakakibara T, Nakamura J, Hotta N: Does cerebral small vessel disease predict future decline of cognitive function in elderly patients with type 2 diabetes? Diabetes Res Clin Pract 2011;94:91-99.

14 Tombaugh TN, McIntyre NJ: The Mini-Mental State Examination. A comprehensive review. J Am Geriatr Soc 1992;40:922-935.

15 Mohs RC, Rosen WG, Davis KL: The Alzheimer's Disease Assessment Scale: an instrument for assessing treatment efficacy. Psychopharmacol Bull 1983;19:448-450.

16 Shinagawa F, Kobayashi S, Fujita K, Maekawa H: A Japanese Version of the WAIS-R (in Japanese). Tokyo, Nihon Bunka Kagaku-sha, 1990, pp 115-118.

17 Stroop JR: Studies of interference in serial verbal reactions. J Exp Psychol 1935;18:643-662.

18 Yesavage JA: The use of self-rating depression scales in the elderly; in Poon LW (ed): Clinical Memory Assessment of Older Adults. Washington, American Psychological Association, 1986, pp 213-217.

19 Fazekas F, Chawluk JB, Alavi A, Hurtig HI, Zimmerman RA: MR signal abnormalities at 1.5 T in Alzheimer's dementia and normal aging. AJR Am J Roentgenol 1987;149:351-356.

-20 Matsuda H, Mizumura S, Nemoto K, Yamashita F, Imabayashi E, Sato N, Asada T: Automatic voxel-based morphometry of structural MRI by SPM8 plus diffeomorphic anatomic registration through exponentiated Lie algebra improves the diagnosis of probable Alzheimer disease. AJNR Am J Neuroradiol 2012;33:1109-1114.

-21 Kashiwagi A, Kasuga M, Araki E, Oka Y, Hanafusa T, Ito H, Tominaga M, Oikawa S, Noda M, Kawamura T, Sanke T, Namba M, Hashimoto M, Sasahara T, Nishio Y, Kuwa K, Ueki K, Takei I, Umemoto M, Murakami Y, Ohashi H: International clinical harmonization of glycated hemoglobin in Japan: from Japan Diabetes Society to National Glycohemoglobin Standardization Program values. J Diabetes Investig 2012;3:39-40.

-22 Kawamura T, Umemura T, Kanai A, Nagashima M, Nakamura N, Uno T, Nakayama M, Sano T, Hamada Y, Nakamura J, Hotta N: Soluble adhesion molecules and C-reactive protein in the progression of silent cerebral infarction in patients with type 2 diabetes mellitus. Metabolism 2006;55:461-466.

23 Nghiem NT, Mai TTT, Ohmori R, Kuroki M, van Chuyen N, Hung NTK, Kawakami M, Kondo K: Apolipoprotein E polymorphism in Vietnamese children and its relationship to plasma lipid and lipoprotein levels. Metabolism 2004;53:1517-1521.

-24 Matsuo S, Imai E, Horio M, Yasuda Y, Tomita K, Nitta K, Yamagata K, Tomino Y, Yokoyama H, Hishida A: Revised equations for estimated GFR from serum creatinine in Japan. Am J Kidney Dis 2009;53:982-992.

25 de Bresser J, Tiehuis AM, van den Berg E, Reijmer YD, Jongen C, Kappelle LJ, Mali WP, Viergever MA, Biessels GJ: Progression of cerebral atrophy and white matter hyperintensities in patients with type 2 diabetes. Diabetes Care 2010;33:1309-1314.

-26 Willette AA, Xu G, Johnson SC, Birdsill AC, Jonaitis EM, Sager MA, Hermann BP, La Rue A, Asthana S, Bendlin BB: Insulin resistance, brain atrophy, and cognitive performance in late middle-aged adults. Diabetes Care 2013;36:443-449.

27 Cherbuim N, Sachdev P, Anstey KJ: High normal fasting plasma glucose is associated with hippocampal atrophy. The PATH Study. Neurology 2012;79:1019-1026.

28 Kerti L, Witte AV, Winkler A, Grittner U, Rujescu D, Flöel A: Higher glucose levels associated with lower memory and reduced hippocampal microstructure. Neurology 2013;81:1746-1752.

29 Launer LJ, Miller M, Williamson JD, Lazar RM, Gerstein HC, Murray AM, Sullivan MD, Horowitz KR, Ding J, Marcovina S, Lovato LC, Lovato J, Margolis KL, O’Connor P, Lipkin EW, Hirsch J, Coker LH, Maldjian J, Sunshine JL, Truwit C, Davatzikos C, Bryan RN: Effects of intensive glucose lowering on brain structure and function in people with type 2 diabetes (ACCORD MIND): a randomized open-label substudy. Lancet Neurol 2011;10:969-977. 
-30 Geijselaers SL, Sep SJ, Stehouwer CD, Biessels GJ: Glucose regulation, cognition, and brain MRI in type 2 diabetes: a systematic review. Lancet Diabetes Endocrinol 2015;3:75-89.

-31 Sink KM, Divers J, Whitlow CT, Palmer ND, Smith SC, Xu J, Hugenschmidt CE, Wagner BC, Williamson JD, Bowden DW, Maldjian JA, Freedman BI: Cerebral structural changes in diabetic kidney disease: African American-Diabetes Heart Study MIND. Diabetes Care 2015;38:206-212.

-32 Mehta D, Pimentel DA, Núñez M-Z, Abduljalil A, Novak V: Subclinical albuminuria is linked to gray matter atrophy in type 2 diabetes mellitus. Metabolism 2014;63:1390-1397.

-33 Knopman DS, Mosley TH Jr, Bailey KR, Jack CR Jr, Schwartz GL, Turner ST: Associations of microalbuminuria with brain atrophy and white matter hyperintensities in hypertensive sibships. J Neurol Sci 2008;271:53-60.

34 Vogels SCM, Emmelot-Vonk MH, Verhaar HJJ, Koek HL: The association of chronic kidney disease with brain lesions on MRI or CT: a systematic review. Maturitas 2012;71:331-336.

-35 de Bresser J, Reijmer YD, van den Berg E, Breedijk MA, Kappelle LJ, Viergever MA, Biessels GJ: Microvascular determinants of cognitive decline and brain volume change in elderly patients with type 2 diabetes. Dement Geriatr Cogn Disord 2010;30:381-386.

36 Murray AM: The brain and the kidney connection: a model of accelerated vascular cognitive impairment. Neurology 2009;73:916-917.

-37 Zlokovic BV: Neurovascular pathways to neurodegeneration in Alzheimer's disease and other disorders. Nat Rev Neurosci 2011;12:723-738.

-38 Barzilay JI, Lovato JF, Murray AM, Williamson J, Ismail-Beigi F, Karl D, Papademetriou V, Launer LJ: Albuminuria and cognitive decline in people with diabetes and normal renal function. Clin J Am Soc Nephrol 2013; 8:1907-1914.

39 Umemura T, Kawamura T, Umegaki H, Kawano N, Mashita S, Sakakibara T, Hotta N, Sobue G: Association of chronic kidney disease and cerebral small vessel disease with cognitive impairment in elderly patients with type 2 diabetes. Dement Geriatr Cogn Disord Extra 2013;3:212-222.

-40 Kawamura T, Umemura T, Umegaki H, Imamine R, Kawano N, Tanaka C, Kawai M, Minatoguchi M, Kusama M, Kouchi Y, Watarai A, Kanai A, Nakashima E, Hotta N: Effect of renal impairment on cognitive function during a 3-year follow up in elderly patients with type 2 diabetes: association with microinflammation. J Diabetes Investig 2014;5:597-605.

41 Umemura T, Kawamura T, Umegaki H, Mashita S, Kanai A, Sakakibara T, Hotta N, Sobue G: Endothelial and inflammatory markers in relation to progression of ischaemic cerebral small-vessel disease and cognitive impairment: a 6-year longitudinal study in patients with type 2 diabetes mellitus. J Neurol Neurosurg Psychiatry 2011;82:1186-1194.

42 Appelman APA, Exalto LG, van der Graaf Y, Biessels GJ, Mali WPTM, Geerlings MI: White matter lesions and brain atrophy: more than shared risk factors? A systematic review. Cerebrovasc Dis 2009;28:227-242.

-43 Kloppenborg RP, Nederkoorn PJ, Grool AM, Vincken KL, Mali WPTM, Vermeulen M, van der Graaf Y, Geerlings MI: Cerebral small-vessel disease and progression of brain atrophy: the SMART-MR study. Neurology 2012; 79:2029-2036.

44 Moran C, Phan TG, Chen J, Blizzard L, Beare R, Venn A, Münch G, Wood AG, Forbes J, Greenaway TM, Pearson $\mathrm{S}$, Srikanth V: Brain atrophy in type 2 diabetes: regional distribution and influence on cognition. Diabetes Care 2013;36:4036-4042.

45 van Elderen SGC, de Roos A, de Craen AJM, Westendorp RGJ, Blauw GJ, Jukema JW, Bollen ELEM, Middelkoop HAM, van Buchem MA, van der Grond J: Progression of brain atrophy and cognitive decline in diabetes mellitus: a 3-year follow-up. Neurology 2010;75:997-1002. 